\title{
ON THE IRREDUCIBILITY OF HECKE POLYNOMIALS
}

\author{
SCOTT AHLGREN
}

\begin{abstract}
Let $T_{n, k}(X)$ be the characteristic polynomial of the $n$th Hecke operator acting on the space of cusp forms of weight $k$ for the full modular group. We record a simple criterion which can be used to check the irreducibility of the polynomials $T_{n, k}(X)$. Using this criterion with some machine computation, we show that if there exists $n \geq 2$ such that $T_{n, k}(X)$ is irreducible and has the full symmetric group as Galois group, then the same is true of $T_{p, k}(X)$ for each prime $p \leq 4,000,000$.
\end{abstract}

\section{INTRODUCTION AND STATEMENT OF RESUltS}

Let $k$ be a positive even integer, and let $S_{k}$ denote the space of cusp forms of weight $k$ for the modular group $\mathrm{SL}_{2}(\mathbb{Z})$. With $q:=e^{2 \pi i z}$, we may identify a cusp form $f(z) \in S_{k}$ with its Fourier expansion

$$
f(z)=\sum_{n=1}^{\infty} a_{n}(f) q^{n} .
$$

For each prime $p$, the Hecke operator $T_{p, k}$ is an endomorphism of $S_{k}$ whose action is described via the formula

$$
T_{p, k}\left(\sum_{n=1}^{\infty} a_{n} q^{n}\right)=\sum_{n=1}^{\infty}\left(a_{n p}+p^{k-1} a_{n / p}\right) q^{n} .
$$

More generally, for $n \geq 1$, the operators $T_{n}$ are defined via the Euler product

$$
\sum T_{n, k} n^{-s}=\prod_{p}\left(1-T_{p, k} p^{-s}+p^{k-1-2 s}\right)^{-1} .
$$

For complete background one may consult [7, for example.

We let $T_{n, k}(X)$ be the characteristic polynomial of the operator $T_{n, k}$ on the space $S_{k}$. Since $S_{k}$ has a basis of forms with rational integer coefficients, we see from (1.1) and (1.2) that $T_{n, k}(X) \in \mathbb{Z}[X]$. Let $d_{k}$ denote the dimension of the complex vector space $S_{k}$, and let $\Omega_{d}$ denote the symmetric group on $d$ letters. Maeda ([5], Conjecture 1.2) made the conjecture that the Hecke algebra of each $S_{k}$ over $\mathbb{Q}$ is simple (i.e. it is a single number field) and that the Galois closure has Galois group $\Omega_{d_{k}}$. This would be implied by the existence of an $n$ for which $T_{n, k}(X)$ is irreducible with group $\Omega_{d_{k}}$. The conjecture implies that there is a single Galois

Received by the editor February 21, 2007 and, in revised form, May 31, 2007.

2000 Mathematics Subject Classification. Primary $11 \mathrm{~F} 11$.

The author thanks the National Science Foundation for its support through grant DMS 0134577. He also thanks the Department of Computing at Macquarie University for its hospitality during part of the time when this research was conducted.

(C) 2008 American Mathematical Society
Reverts to public domain 28 years from publication 
orbit of Hecke eigenforms in $S_{k}$, which has implications for the work of Maeda and Hida [5] on base change for totally real fields.

In recent years a number of authors have investigated the irreducibility of the polynomials $T_{n, k}(X)$. James and Ono [6] studied the reductions of Hecke polynomials (of arbitrary level) modulo primes $\ell$. Buzzard 2] proved that if $\ell \leq 19$ is prime and $k=12 \ell$, then the polynomial $T_{2, k}$ is irreducible and has Galois group $\Omega_{d_{k}}$. Exploiting some periodicities in the factorization of $T_{p, k}(X)$ modulo 5 and 7 , Conrey, Farmer, and Wallace proved the following result.

Theorem 1.1 ([3], Theorem 1). Let $k$ be a positive even integer, and suppose that there exists an $n$ for which $T_{n, k}(X)$ is irreducible and has Galois group $\Omega_{d_{k}}$. Then the same is true of the polynomial $T_{p, k}(X)$ for each prime $p$ such that

$$
p \not \equiv \pm 1 \quad(\bmod 5) \quad \text { or } \quad p \not \equiv \pm 1 \quad(\bmod 7) .
$$

Farmer and James [4] proved that for each $p \leq 2000$ and each $k \leq 2000$, the polynomial $T_{p, k}(X)$ is irreducible and has Galois group $\Omega_{d_{k}}$. More recently, Baba and Murty, using Frobenius distributions and an effective form of the Chebotarev Density Theorem, have proved the following.

Theorem 1.2 (1], Theorem 1.1). Let $k$ be a positive even integer, and suppose that there exists a prime $p$ for which $T_{p, k}(X)$ is irreducible. Then for some $\delta>0$ we have

$$
\#\left\{p \leq N: T_{p, k}(X) \text { is reducible }\right\} \ll \frac{N}{(\log N)^{1+\delta}} .
$$

Baba and Murty also obtain

Theorem 1.3 (1), Theorem 1.2). Let $k$ be a positive even integer, and suppose that there exists a prime $p$ for which $T_{p, k}(X)$ is irreducible with Galois group $\Omega_{d_{k}}$. Then the same holds for $T_{n, k}(X)$ for all $n \leq d_{k}$.

Our aim here is to record a simple criterion which is equivalent to the irreducibility of $T_{n, k}(X)$ under the hypotheses in Theorems 1.1, 1.2, and 1.3, This criterion is quite amenable to computation (cf. Theorem 1.5 below). To simplify the exposition, we introduce "Hypothesis O" for the space $S_{k}$ :

Hypothesis $\mathbf{O}$ for $S_{k}$ : There exists an $n$ for which $T_{n, k}(X)$ is irreducible and has Galois group $\Omega_{d_{k}}$.

We define the space $S_{k}^{\prime}$ by

$$
S_{k}^{\prime}:=\left\{f \in S_{k}: \operatorname{ord}_{\infty}(f) \geq 2\right\} .
$$

When $\operatorname{dim} S_{k} \geq 1$ we have

$$
\operatorname{dim} S_{k}^{\prime}=\operatorname{dim} S_{k}-1 .
$$

With this notation, we have the following result.

Theorem 1.4. Let $k$ be such that $\operatorname{dim} S_{k} \geq 2$, and suppose that $S_{k}$ satisfies $H y$ pothesis $O$. Then for every $n \geq 2$, the following are equivalent:

(1) $T_{n, k}(X)$ is irreducible with Galois group $\Omega_{d_{k}}$.

(2) There exists a modular form $f \in S_{k}^{\prime}$ such that $a_{n}(f) \neq 0$. 
Remark. It is known that the polynomial $T_{2,24}(X)$ is irreducible. Let $\Delta(z)$ be the unique normalized cusp form of weight 12 (defined explicitly in (1.6) below), and write

$$
\Delta^{2}(z)=\sum_{n=2}^{\infty} a(n) q^{n}
$$

Since $d_{24}=2$, it follows from Theorem 1.4 that

$$
T_{n, 24}(X) \text { is irreducible } \Longleftrightarrow a(n) \neq 0 .
$$

One might speculate that the coefficients $a(n)$ in (1.4) are never zero (or more generally that the second condition in Theorem 1.4 is always satisfied). To prove this seems to be a very difficult problem; the same speculation for the coefficients of $\Delta(z)$ is a famous unsolved problem of Lehmer.

It is typically easy to check the second condition in Theorem 1.4 numerically. Via some machine computation, we obtain the following result.

Theorem 1.5. Let $k$ be such that $\operatorname{dim} S_{k} \geq 2$, and suppose that $S_{k}$ satisfies Hypothesis $O$. Then the following are true:

(1) $T_{p, k}(X)$ is irreducible with Galois group $\Omega_{d_{k}}$ for each prime $p \leq 4,000,000$.

(2) $T_{n, k}(X)$ is irreducible with Galois group $\Omega_{d_{k}}$ for each $n \in\{2,3, \ldots, 10,000\}$.

Remark. This may be compared with Theorem 1.3 when $k$ is large. The bounds in Theorem 1.5 could easily be increased if necessary with more computation.

We briefly indicate the connection between Theorem 1.1 and Theorem 1.4. To this end, we recall the definition of the Eisenstein series

$$
E_{k}(z):=1-\frac{2 k}{B_{k}} \sum_{n=1}^{\infty} \sigma_{k-1}(n) q^{n},
$$

where $k \geq 4$ is even, $B_{k}$ is the $k$ th Bernoulli number, and $\sigma_{k-1}(n)$ is the sum of the $(k-1)$ st powers of the divisors of $n$. Each $E_{k}$ is a modular form of weight $k$ for $\mathrm{SL}_{2}(\mathbb{Z})$. For primes $p \geq 5$, the von Staudt-Claussen theorem on the $p$-adic valuation of Bernoulli numbers implies the congruence

$$
E_{p-1}(z) \equiv 1 \quad(\bmod p) .
$$

We have the relation

$$
\Delta(z):=\frac{E_{4}^{3}(z)-E_{6}^{2}(z)}{1728} .
$$

We have the following congruences, which can be verified using standard techniques from the theory of modular forms modulo $\ell$ (see, for example, [8] or [7]):

$$
\begin{aligned}
\Delta^{2} & \equiv 2 \sum n\left(n^{2}-1\right) \sigma(n) q^{n} \quad(\bmod 5), \\
\Delta^{2} E_{6} & \equiv \sum n^{2}\left(n^{2}-1\right) \sigma(n) q^{n} \quad(\bmod 5),
\end{aligned}
$$

and

$$
\begin{aligned}
\Delta^{2} & \equiv \sum\left(n^{2} \sigma(n)-n \sigma_{3}(n)\right) q^{n} \quad(\bmod 7), \\
\Delta^{2} E_{4} & \equiv 2 \sum\left(n \sigma(n)-n^{3} \sigma_{3}(n)\right) q^{n} \quad(\bmod 7), \\
\Delta^{2} E_{4}^{2} & \equiv 3 \sum\left(n^{2} \sigma_{3}(n)-n^{3} \sigma(n)\right) q^{n} \quad(\bmod 7) .
\end{aligned}
$$


In view of (1.5), we see that if $\operatorname{dim} S_{k} \geq 2$, then the space $S_{k}^{\prime}$ contains a modular form with integer coefficients which is congruent to one of the two forms in (1.7) modulo 5, and a form which is congruent to one of the three forms in (1.8) modulo 7. Therefore we obtain the following as corollaries to Theorem 1.4.

Corollary 1.6. Suppose that $\operatorname{dim} S_{k} \geq 2$ and that $S_{k}$ has Hypothesis O. Suppose that

$$
n\left(n^{2}-1\right) \sigma(n) \not \equiv 0 \quad(\bmod 5) .
$$

Then $T_{n, k}(X)$ is irreducible and has Galois group $\Omega_{d_{k}}$.

Corollary 1.7. Suppose that $\operatorname{dim} S_{k} \geq 2$ and that $S_{k}$ has Hypothesis O. Suppose that

$$
\begin{array}{lll}
n^{2} \sigma(n)-n \sigma_{3}(n) \not \equiv 0 & (\bmod 7) & \text { if } k \equiv 0,2 \quad(\bmod 6), \\
n \sigma(n)-n^{3} \sigma_{3}(n) \not \equiv 0 & (\bmod 7) & \text { if } k \equiv 4 \quad(\bmod 6) .
\end{array}
$$

Then $T_{n, k}(X)$ is irreducible and has Galois group $\Omega_{d_{k}}$.

Specializing to the case when $n=p$ is prime, we see that Theorem 1.1 follows from these corollaries.

\section{Proof of Theorem 1.4}

The proof is similar to that of Theorem [1.3. We begin with a lemma which has been recorded in various forms in [3, 4, and 1]. We give a proof here for completeness.

Lemma 2.1. Suppose that $k \geq 2$ is an even integer and that there exists $n_{0} \geq 2$ with the property that $T_{n_{0}, k}(X)$ is irreducible with Galois group $\Omega_{d_{k}}$. Then for each $n \geq 2$, one of the following is true:

(1) $T_{n, k}(X)$ is irreducible.

(2) $T_{n, k}(X)=(X-a)^{d_{k}}$ for some constant a.

In the first case, $T_{n, k}(X)$ has Galois group $\Omega_{d_{k}}$.

Proof. Set $d=d_{k}$ and let

$$
\left\{f_{1}, \ldots, f_{d}\right\}
$$

be a basis of normalized eigenforms for the space $S_{k}$. Let the Fourier expansions of these forms be given by

$$
\begin{array}{cc}
f_{1}=q+\cdots+ & a_{n_{0}}\left(f_{1}\right) q^{n_{0}}+\cdots+a_{n}\left(f_{1}\right) q^{n}+\ldots \\
\vdots & \vdots \\
f_{d}=q+\cdots+ & a_{n_{0}}\left(f_{d}\right) q^{n_{0}}+\cdots+a_{n}\left(f_{d}\right) q^{n}+\ldots
\end{array}
$$

Then for each $n$ we have

$$
T_{n, k}(X)=\left(X-a_{n}\left(f_{1}\right)\right) \ldots\left(X-a_{n}\left(f_{d}\right)\right) .
$$

By hypothesis, the roots $a_{n_{0}}\left(f_{i}\right)$ are distinct and the Galois group $\operatorname{Gal}(\overline{\mathbb{Q}} / \mathbb{Q})$ acts as the full permutation group $\Omega_{d}$ on the set $\left\{a_{n_{0}}\left(f_{i}\right)\right\}$. It follows that $\operatorname{Gal}(\overline{\mathbb{Q}} / \mathbb{Q})$ acts as the full permutation group $\Omega_{d}$ on $\left\{f_{i}\right\}$ and therefore that it acts transitively on $\left\{a_{n}\left(f_{i}\right)\right\}$. From this we see that $T_{n, k}(X)=f(X)^{r}$ for some $r$, where $f(X)$ is an irreducible polynomial of degree $e:=d / r$. If it were the case that both $e>1$ and $r>1$, then we could select distinct indices $i, j$, and $k$ for which $a_{n}\left(f_{i}\right)=a_{n}\left(f_{j}\right)$ and 
$a_{n}\left(f_{i}\right) \neq a_{n}\left(f_{k}\right)$. However, this would contradict the fact that $\operatorname{Gal}(\overline{\mathbb{Q}} / \mathbb{Q})$ contains an element $\sigma$ whose action on $\left\{f_{i}\right\}$ is described by the cycle $(i, j, k)$. Therefore either $T_{n, k}(X)$ is irreducible or has the form $(X-a)^{d}$. In the former case it is clear that $\mathrm{Gal}(\overline{\mathbb{Q}} / \mathbb{Q})$ acts as the full permutation group on the set of roots.

Proof of Theorem 1.4. Let $k$ be as in the hypotheses. Suppose first that $n \geq 2$ is an integer for which $T_{n, k}(X)$ is reducible; by Lemma 2.1] it follows that there is a constant $a$ with the property that $a_{n}\left(f_{i}\right)=a$ for each eigenform $f_{i}$ in the basis (2.1). Let $f$ be a form in $S_{k}^{\prime}$, and write

$$
f=\sum_{i=1}^{d} c_{i} f_{i}
$$

as a linear combination of this basis. Since each $f_{i}$ has the form $f_{i}=q+\ldots$, and $f$ vanishes to order $\geq 2$ at infinity, it follows that $\sum c_{i}=0$. But then $a_{n}(f)=$ $\sum c_{i} a=0$.

Conversely, suppose that $n \geq 2$ is such that each form $f \in S_{k}^{\prime}$ has $a_{n}(f)=0$. Writing $f$ as in (2.4), and with notation as in (2.2), we conclude that

$$
\sum_{i=1}^{d} c_{i}=0 \quad \Longrightarrow \quad \sum_{i=1}^{d} c_{i} a_{n}\left(f_{i}\right)=0 .
$$

Therefore there exists a constant $a$ with $a_{n}\left(f_{i}\right)=a$ for all $i$, and so $T_{n, k}(X)=$ $(X-a)^{d}$.

\section{Proof of Theorem 1.5}

To prove Theorem 1.5. we use Theorem[1.4 together with some machine computation. Write $S_{k}^{\prime}(\mathbb{Z})$ for the $\mathbb{Z}$-module of forms in $S_{k}^{\prime}$ with rational integer coefficients. Recall the congruence (1.5). To prove Theorem [1.5) it will suffice, for each prime $p<4,000,000$ and for each $k$ with $\operatorname{dim} S_{k} \geq 2$, to produce the following:

(1) A prime $\ell \geq 5$ and an integer $k_{0} \leq k$ with $k_{0} \equiv k(\bmod \ell-1)$.

(2) A form $f \in S_{k_{0}}^{\prime}(\mathbb{Z})$ with the property that $a_{p}(f) \not \equiv 0(\bmod \ell)$.

Define the Eisenstein series $\widetilde{E}_{k}$ via the formulas

$$
\widetilde{E}_{k}:=\left\{\begin{array}{lll}
1 & \text { if } k \equiv 0 \quad(\bmod 12), \\
E_{14} & \text { if } k \equiv 2 \quad(\bmod 12), \\
E_{4} & \text { if } k \equiv 4 \quad(\bmod 12), \\
E_{6} & \text { if } k \equiv 6 \quad(\bmod 12), \\
E_{8} & \text { if } k \equiv 8 \quad(\bmod 12), \\
E_{10} & \text { if } k \equiv 10 \quad(\bmod 12) .
\end{array}\right.
$$

Suppose first that $k \geq 240$ and $k \neq 242$; in this case the computations can be done using the primes $\ell=5$ and $\ell=7$. We fix a class $\bar{k}(\bmod 12)$ of weights and compute, for each $d \in\{2,3, \ldots, 20\}$, the first $4,000,000$ coefficients of each of the modular forms

$$
f_{d, 5}:=\Delta^{d} \widetilde{E}_{\bar{k}} \quad(\bmod 5) \quad \text { and } \quad f_{d, 7}:=\Delta^{d} \widetilde{E}_{\bar{k}} \quad(\bmod 7) .
$$


To simplify computations, we use the formulas

$$
\Delta \equiv \sum_{n=1}^{\infty} n \sigma_{1}(n) \quad(\bmod 5), \quad \Delta \equiv \sum_{n=1}^{\infty} n \sigma_{3}(n) \quad(\bmod 7) .
$$

We find, for each $p \leq 4,000,000$, that at least one of the forms $f_{d, \ell}$ in (3.2) has the property that

$$
a_{p}\left(f_{d, \ell}\right) \not \equiv 0 \quad(\bmod \ell) .
$$

This establishes the result when $k \geq 240, k \neq 242$. We note that this step required a little more than one hour of computation (using MAGMA) on a desktop computer.

The weights $k<240$ and $k=242$ can be dealt with individually (in several hours of computation). In view of congruences like (1.7) and (1.8), small primes $\ell$ are less suitable for this purpose. To proceed, we compute, for each $2 \leq d \leq 19$, and for each residue class $\bar{k}(\bmod 12)$ of weights, the first 4, 000, 000 coefficients of each of the modular forms

$$
g_{(d, \bar{k}), 691}:=\Delta^{d} \widetilde{E_{\bar{k}}} \quad(\bmod 691) \quad \text { and } \quad g_{(d, \bar{k}), 701}:=\Delta^{d} \widetilde{E}_{\bar{k}} \quad(\bmod 701)
$$

(these particular primes are used for the sole reason that their size is suited to the purpose). For each pair $(d, \bar{k})$, we see from this computation that for almost every prime $p$ with $d \leq p<4,000,000$, at least one of the following is true:

(1) The $p t h$ coefficient of $g_{(d, \bar{k}), 691}$ is non-zero modulo 691.

(2) The $p$ th coefficient of $g_{(d, \bar{k}), 701}$ is non-zero modulo 701 .

The exceptional primes $p_{1}, \ldots, p_{e}$ are those for which neither of the two conditions hold (for none of the pairs $(d, \bar{k})$ in our range are there more than 3 exceptional primes). Most of these can be eliminated using Theorem 1.1, The few remaining exceptional primes $p$ can be eliminated by exhibiting another modular form $h \in S_{k}^{\prime}$ with the property that $a_{p}(h) \not \equiv 0(\bmod 7)$. To illustrate, we record the result of this computation for those weights $k<240$ with $k \equiv 10(\bmod 12)$.

Exceptional primes when $\bar{k}=10,2 \leq d \leq 19$.

\begin{tabular}{|c|c|c|}
\hline$d$ & Exceptional primes $p$ & Reason for elimination \\
\hline 3 & 1337363 & $p \equiv 3(\bmod 5)$ \\
\hline 4 & 3906883 & $p \equiv 3(\bmod 5)$ \\
\hline 9 & 3911 & $p \equiv 5(\bmod 7)$ \\
\hline \multirow{2}{*}{11} & 718411 & $p$ th coeff. of $\Delta^{4} E_{4} E_{6}^{15}$ is $3(\bmod 7)$ \\
& 3565921 & $p \equiv 2(\bmod 7)$ \\
\hline 13 & 189671 & $p$ th coeff. of $\Delta^{4} E_{4} E_{6}^{19}$ is $2(\bmod 7)$ \\
\hline 14 & 80777 & $p \equiv 2(\bmod 5)$ \\
\hline 15 & 902263 & $p \equiv 3(\bmod 5)$ \\
\hline 18 & 3019571 & $p \equiv 2(\bmod 7)$ \\
\hline
\end{tabular}

Similar methods can be used to establish the second assertion in Theorem 1.5 for brevity we do not include the details here. 


\section{REFERENCES}

[1] S. Baba and M. R. Murty, Irreducibility of Hecke polynomials, Math. Res. Lett. 10 (2003), no. 5-6, 709-715. MR2024727 (2005g:11064)

[2] K. Buzzard, On the eigenvalues of the Hecke operator $T_{2}$, J. Number Theory $\mathbf{5 7}$ (1996), no. 1, 130-132. MR 1378578 (96m:11033)

[3] J. B. Conrey, D. W. Farmer and P. J. Wallace, Factoring Hecke polynomials modulo a prime, Pacific J. Math. 196 (2000), no. 1, 123-130. MR1797238(2001k:11072)

[4] D. W. Farmer and K. James, The irreducibility of some level 1 Hecke polynomials, Math. Comp. 71 (2002), no. 239, 1263-1270. MR.1898755 (2003e:11046)

[5] H. Hida and Y. Maeda, Non-abelian base change for totally real fields, Pacific J. Math. 1997, Special Issue, 189-217. MR1610859 (99f:11068)

[6] K. James and K. Ono, A note on the irreducibility of Hecke polynomials, J. Number Theory 73 (1998), no. 2, 527-532. MR.1658012 (2000a:11063)

[7] S. Lang, Introduction to modular forms, Corrected reprint of the 1976 original, Springer, Berlin, 1995. MR1363488 (96g:11037)

[8] H. P. F. Swinnerton-Dyer, On $l$-adic representations and congruences for coefficients of modular forms, in Modular functions of one variable, III (Proc. Internat. Summer School, Univ. Antwerp, 1972), 1-55. Lecture Notes in Math., 350, Springer, Berlin. MR0406931(53:10717a)

Department of Mathematics, University of Illinois, Urbana, Illinois 61801

E-mail address: ahlgren@math.uiuc.edu 